\title{
Compton Scattered X-Gamma Rays with Orbital Momentum
}

\author{
V. Petrillo, ${ }^{1,2}$ G. Dattoli, ${ }^{3}$ I. Drebot, ${ }^{2}$ and F. Nguyen ${ }^{3}$ \\ ${ }^{1}$ Università degli Studi di Milano, via Celoria 16, 20133 Milano, Italy \\ ${ }^{2}$ INFN-Sezione di Milano, via Celoria 16, 20133 Milano, Italy \\ ${ }^{3}$ ENEA C.R. Frascati, Via E. Fermi, 4500044 Frascati, Roma, Italy
}

(Received 8 July 2016; published 16 September 2016)

\begin{abstract}
We study the possibility of producing x-gamma rays with orbital angular momentum by means of the inverse Compton backscattering between a high brightness electron beam and a twisted laser pulse. We use the classical electrodynamics retarded fields for evaluating the orbital angular momentum of the radiation and connecting it to that of the primary laser pulse. We then propose the dimensioning of a linearly polarized x-ray source with orbital angular momentum, starting from the parameters of operating Thomson setups.
\end{abstract}

DOI: 10.1103/PhysRevLett.117.123903

Optical pulses carrying orbital angular momentum (referred to as OAM beams) are extremely interesting both from the fundamental point of view and for applications. Helically phased light beams or optical vortices, in the visible and infrared wavelength domains, have orbital angular momentum [1] directly transferable to atoms, molecules, and nanostructures [2-7], allowing applications as optical data transmission [8-10], crystal micromanipulation [11], microscopy [12], spectroscopy [13], detection of spinning terrestrial and astronomical objects [14,15], and induction of controlled rotational motions in free electrons [16]. At shorter wavelengths one can envision to employ OAM beams in photoionization experiments, where the violation of the dipolar selection rules could excite new effects [17], in x-ray magnetic circular dichroism, where quadrupolar and dipolar transitions could be unfolded [18], and in the resonant inelastic x-ray scattering of many materials, where the molecules with vibrational degrees of freedom could resonate in the vortex, providing information on their structure and properties [19].

The usual methods for generating OAM beams in the visible range consist of sending light through a fork hologram or a spiral phase mask, achieving efficiencies larger than $40 \%$ [20]. These techniques has also been tried for producing XUV or X-ray OAM beams [21,22] with difficulties and limitations associated to the damage threshold of the optical elements of the beam line at large intensity and to the fabrication of high quality optical surfaces. Therefore, the generation of OAM pulses at short wavelengths and high brilliance is still an open problem.

It has been predicted [23] and then demonstrated experimentally [24] that OAM can be obtained in the regime of spontaneous emission radiation from undulators with variable polarization. Other approaches aimed at generating vortex beams at short wavelengths with freeelectron lasers have been proposed. In a first scheme, a seeded electron beam is transported in a helical undulator [25]. A proof-of-principle test has been recently performed by using a single undulator section, showing the occurrence of optical vortices at $800 \mathrm{~nm}$ [26]. The radiation is produced at the same frequency of the seed, limiting the generation of OAM beams to the operation range of atomic lasers. Another idea takes advantage of the Echo-Enabled Harmonic Generation, where two seed lasers and two magnetic chicanes are used to encode corkscrewlike harmonic microbunching on the electrons [27]. Other proposals foresee the use of seeded higher harmonic generation schemes in the VUV soft x-ray region [28]. Finally, the eigenfunction expression for single photons carrying orbital angular momentum has been constructed [29-31] and the QED cross section for the Compton scattering in these conditions has been analyzed [31].

In this Letter, we study the possibility of producing an OAM x-gamma ray pulse by means of Thomson or inverse Compton backscattering between a relativistic high brightness electron beam and a OAM laser pulse. Using the retarded fields of the classical electrodynamics, we evaluate the optical vector of the radiation from a relativistic electron beam interacting with OAM lasers and we show that the offspring x-ray emission carries an orbital angular momentum connected to that of the primary laser pulse. We then propose the dimensioning of an OAM x-ray source, starting from the parameters of setups already operating or in the commissioning phase.

In the classical electrodynamics [32], the retarded radiation field in the far zone approximation is given by

$$
\underline{E}=\int d \underline{x^{\prime}}\left[\mathcal{H}\left(\underline{x}^{\prime}, t_{r}\right) \frac{\underline{n} \times((\underline{n}-\underline{\beta}) \times \underline{\dot{\beta}})}{c(1-\underline{\beta} \cdot \underline{n})^{2}\left|\underline{x}-\underline{x}^{\prime}\right|}\right]_{\mathrm{ret}},
$$

where $\underline{x}^{\prime}=\left(x^{\prime}, y^{\prime}, z^{\prime}\right)$ is the generic position inside the charge distribution, $\mathcal{H}\left(\underline{x}^{\prime}, t_{r}\right)$ is the electron density, $\underline{n}=$ $\left[\left(\underline{x}-\underline{x}^{\prime}\right) /\left(\left|\underline{x}-\underline{x}^{\prime}\right|\right)\right]$ is the direction of the observer at distance $\left|\underline{x}-\underline{x}^{\prime}\right|$ from the charge, and $\underline{\beta}$ and $\dot{\beta}$ are velocity and acceleration of the particles and are calculated at the retarded time $t_{r}=t-\left|\underline{x}-\underline{x}^{\prime}\right| / c$. Supposing a distribution 
of $N$ particles $\mathcal{H}\left(\underline{x}^{\prime}\right)=-e \sum_{j=1}^{N} \delta\left(\underline{x}^{\prime}-\underline{x}_{j}\left(t_{r}\right)\right)$, the customary expression of the Liénard-Wiechert fields are retrieved.

The acceleration of the particles

$\underline{\dot{\beta}}=\frac{-e}{m_{e} c \gamma}\left[\underline{E}_{L}\left(1-\underline{\beta} \cdot \underline{e}_{k}\right)+\underline{\beta} \cdot \underline{E}_{L}\left(\underline{e}_{k}-\underline{\beta}\right)\right]=\underline{T} \cdot \underline{E}_{L}$

is in turn deduced from the electron momentum equation.

The laser electric field $E_{L}$ of Eq. (2), propagating along the direction $\underline{e}_{k}=-\underline{e}_{z}$, is assumed to carry orbital angular momentum and is represented by [33]

$$
\underline{E}_{L}(x, y, z, t)=E_{0} \underline{e}_{y} f(x, y, z+c t) H_{m}(x, y, z),
$$

where

$f(x, y, z+c t)=\frac{w_{0}^{2}}{w_{z}^{2}} g(z+c t) e^{-\left(x^{2}+y^{2} / 2 w_{z}^{2}\right)} e^{i\left(\omega_{L} t+k_{z L} z\right),}$

with $\omega$ and $k_{z}$, respectively, angular frequency and wave number and $g(z+c t)$ an arbitrary function containing the longitudinal amplitude modulation.

$$
H_{m}(x, y, z)=m ! \sum_{r=0}^{[m / 2]} \frac{\alpha^{r} \xi^{m-2 r}}{r !(m-2 r) !}
$$

are then the Hermite-Kampé de Fériet polynomials, with $\xi=1 / w_{0}\left[\left(w_{0} / w_{z}\right)^{2}(x+i \varepsilon y)-\left(x_{0}+i \varepsilon y_{0}\right)\right]$ and $\alpha=$ $i / 2\left(1-\varepsilon^{2}\right) \lambda z / w_{z}^{2}$. In these expressions $\left(x_{0}, y_{0}\right)$ is the center of the vortex, $\varepsilon$ is an integer number named topological charge, and $w_{z}^{2}=w_{0}^{2}\left[1+i\left(z / z_{R}\right)\right], w_{0}$ being the transverse characteristic lengths of the Gaussian host beam, $z_{R}=$ $\pi w_{0}^{2} / \lambda_{L}$ the Rayleigh length, and $\lambda_{L}=2 \pi / k_{L}$ the laser wavelength. $E_{0}$ represents the peak electric field, and, if the longitudinal dependence is Gaussian, can be expressed through the laser energy $W_{\text {tot }}$ and the transverse and longitudinal rms dimensions $w_{0}$ and $\sigma_{z}$ of the laser pulse as $E_{0}=2 \sqrt{W_{\text {tot }}} /\left(w_{0} \sqrt{\sigma_{z} m ! \sqrt{\pi}}\right)$.

Figure 1 shows the real part of the field (3) at different $z$ for $x_{0}=0, y_{0}=0$, and for $m=0,1,2$, respectively, from the first to the third columns. We can note the dipolar $(m=1)$ or quadrupolar $(m=2)$ structure of these last cases and the central zero of the field in correspondence to the position of the vortex.

The proposed OAM x-gamma ray source is sketched in Fig. 2 and the parameter we have used, similar to those of operating Thomson scattering sources [34,35] (Table I, second column), are listed in Table I, third column.

In particular, we consider a ps-long electron beam produced by a photoinjector and accelerated in a linac up to the value of $25 \mathrm{MeV}$, with a charge of $1 \mathrm{nC}$, with transverse dimension $\sigma=100 \mu \mathrm{m}$. The laser beam is a Ti: sapphire high energy device of wavelength $\lambda_{L}=c \omega_{L}=$ $0.8 \mu \mathrm{m}$, focused in the interaction point up to rms values of
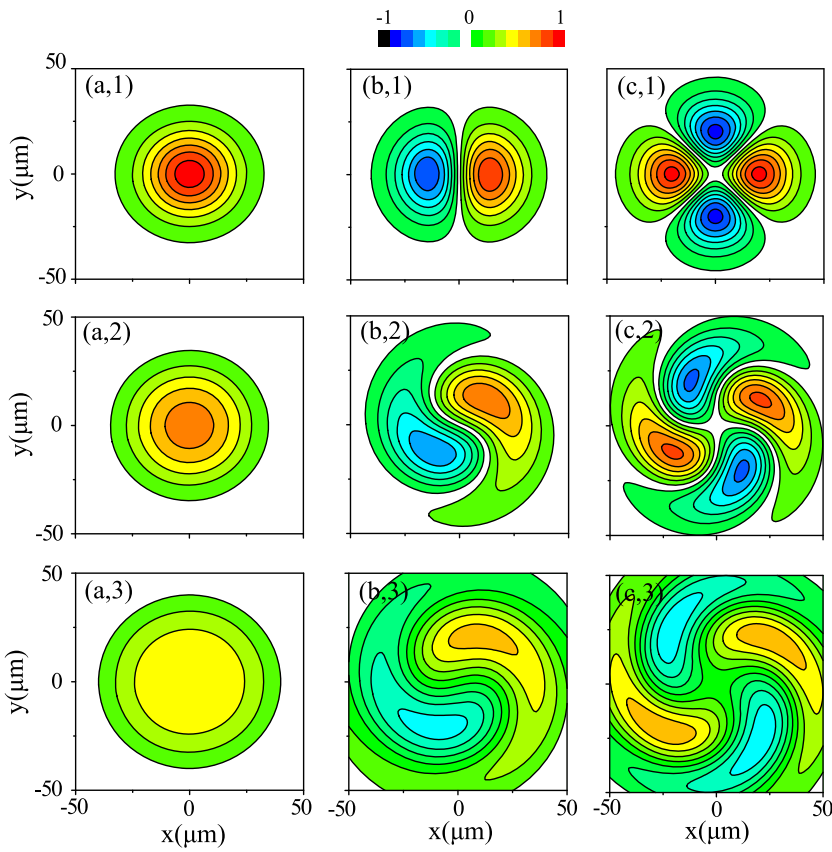

FIG. 1. Laser field structure for $x_{0}=0, y_{0}=0$ and (a) $m=0$, (b) $m=1, \varepsilon=1$; (c) $m=2, \varepsilon=1$, (1) $z=0$, (2) $z=0.001 \mathrm{~mm}$ (3) $z=0.002 \mathrm{~mm}$.

the spot of about $20 \mu \mathrm{m}$ and with a Gaussian temporal structure with rms time duration of about 1 ps. The radiation frequency emitted by one electron [36] maintains the customary expression

$$
\omega=\omega_{L} \frac{1+\underline{\beta} \cdot \underline{e}_{k}}{1-\underline{\beta} \cdot \underline{n}} \approx 4 \omega_{L} \gamma^{2}
$$

giving, in the present case, the radiation photon energy edge at $8 \mathrm{keV}$. The interaction is supposed to be head-on and the parameters fall within the linear regime. Since $4 \hbar \omega_{L} \gamma /\left(m_{e} c^{2}\right) \approx 0.6 \times 10^{-3} \ll 1$, quantum corrections are also negligible. The intensity of the radiation field for a reference Gaussian case $(m=0)$ is shown in Fig. 3. The total number of photons per shot is about $2.5 \times 10^{6}$.

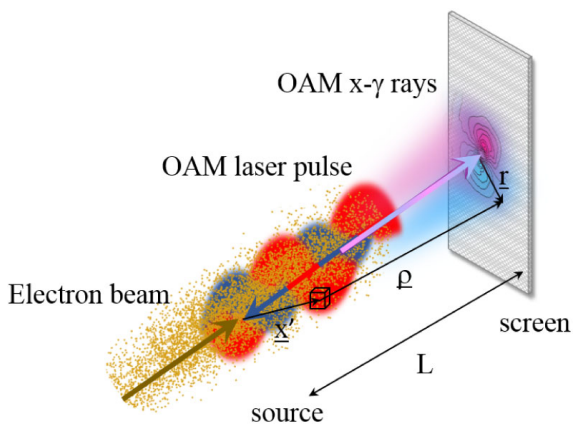

FIG. 2. Scheme and geometry of the OAM x- $\gamma$ source. $L$ : distance between the source and the screen. $\underline{x}^{\prime}$ : position of the generic charge element. $(x, y, z)=(r, \varphi, z)$ : position of the observer. 
TABLE I. Electron and laser parameters. First column: typical values of real Compton sources. Second column: values used in the calculations.

\begin{tabular}{lcc}
\hline \hline Electron energy & $25-50 \mathrm{MeV}$ & $25 \mathrm{MeV}$ \\
Electron charge & $0.25-1 \mathrm{nC}$ & $1 \mathrm{nC}$ \\
Electron radius & $10-100 \mu \mathrm{m}$ & $100 \mu \mathrm{m}$ \\
Electron length & $1-10 \mathrm{~mm}$ & $1 \mathrm{~mm}$ \\
Laser wavelength & $0.8 \mu \mathrm{m}$ & $0.8 \mu \mathrm{m}$ \\
Laser energy & $0.5-5 \mathrm{~J}$ & $1 \mathrm{~J}$ \\
Laser waist & $10-50 \mu \mathrm{m}$ & $20 \mu \mathrm{m}$ \\
Laser duration & $1-6 \mathrm{ps}$ & $1 \mathrm{ps}$ \\
Repetition rate & $10 \mathrm{~Hz}$ & \\
\hline \hline
\end{tabular}

The cases with $m=1$ and $m=2$ are presented in Figs. 4 and 5. The first three windows (a),(b), and (c) show snapshots on the screen of the $y$ component of the radiation field at different times, while the last one represents the temporal average of the intensity. The structure of the time averaged intensity of the electric field replicates the laser's shape, the total photon number is the same as the Gaussian case.

For a linearly polarized field, as is our case both for laser and radiation [37], the $z$ component of the orbital angular momentum density of a monochromatic signal, according to Refs. [25,32], is

$$
\frac{d L_{z}^{O M}}{d V}=\frac{1}{4 \pi} \sum_{k=1}^{3} \frac{E_{k}}{i \omega}\left(x \frac{\partial E_{k}^{*}}{\partial y}-y \frac{\partial E_{k}^{*}}{\partial x}\right) .
$$

For the laser radiation, in the case of $\varepsilon=1$, vortex in the center $\left(x_{0}, y_{0}\right)=(0,0)$, and very long Rayleigh length $\left(z_{R} \gg w_{0}\right)$,

$$
\frac{d L_{L, z}^{\mathrm{OM}}}{d V}=-m \frac{E_{L}^{2}}{4 \pi \omega_{L}}
$$

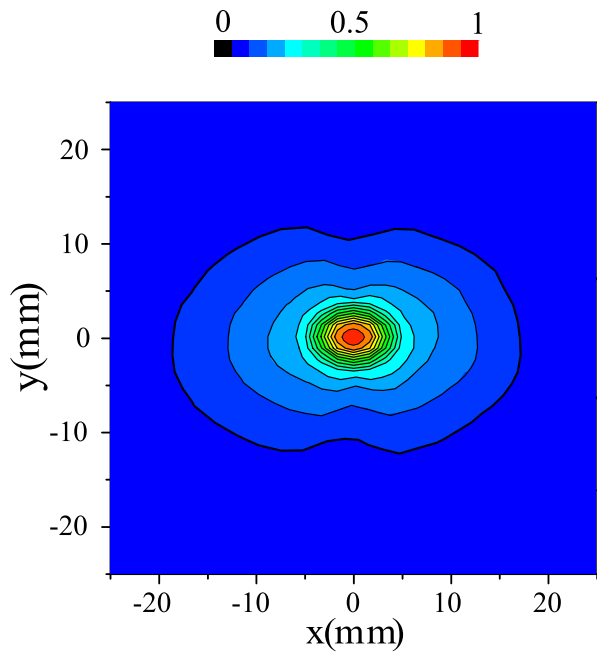

FIG. 3. Radiation average intensity for a reference Gaussian case.
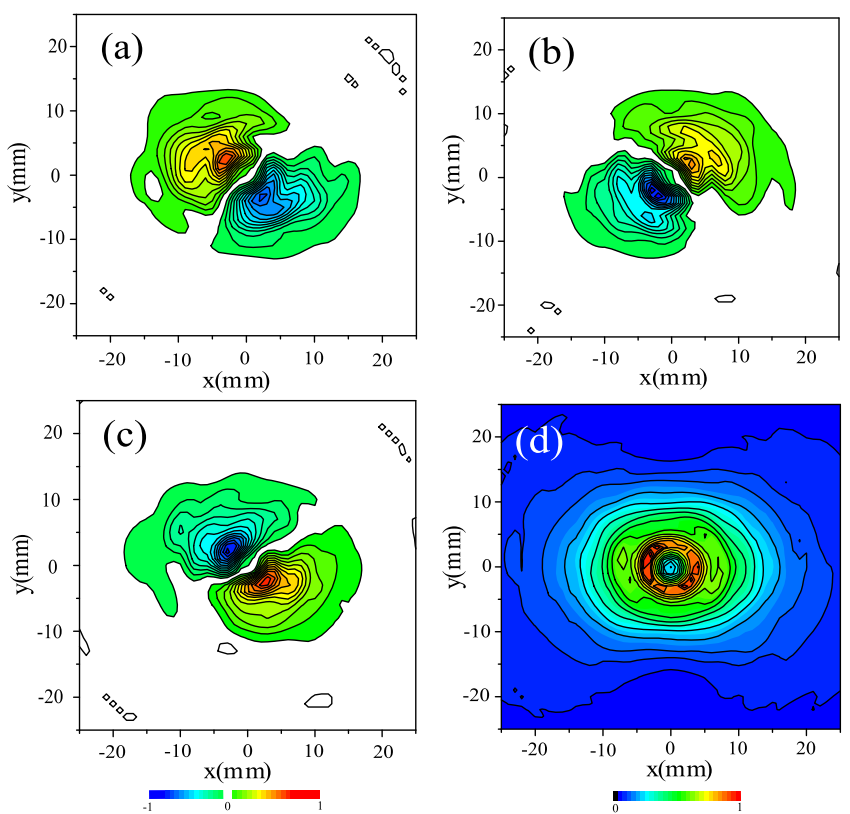

FIG. 4. $y$ component of the electric field $\underline{E}$ of the radiation at different times. (a) $t=0.2$, (b) $t=0.4$, (c) $t=0.6$ ps. (d) average intensity for $m=1, \varepsilon=1$.

Choosing simplified conditions, i.e., a monochromatic beam $\beta_{i}=\beta_{0} \underline{e}_{z}$ with an electron distribution $\mathcal{H}=$ $-e \sum \delta\left(\underline{x}^{\prime}-\underline{x}_{j}\left(t_{r}\right)\right)$ over a volume whose transverse dimension is larger than $w_{0}$, and considering only the dominant component $y$ of the radiation, we can show that there is a direct link between the orbital angular momentum of the laser and the orbital angular momentum of the radiation. In this case, in fact,
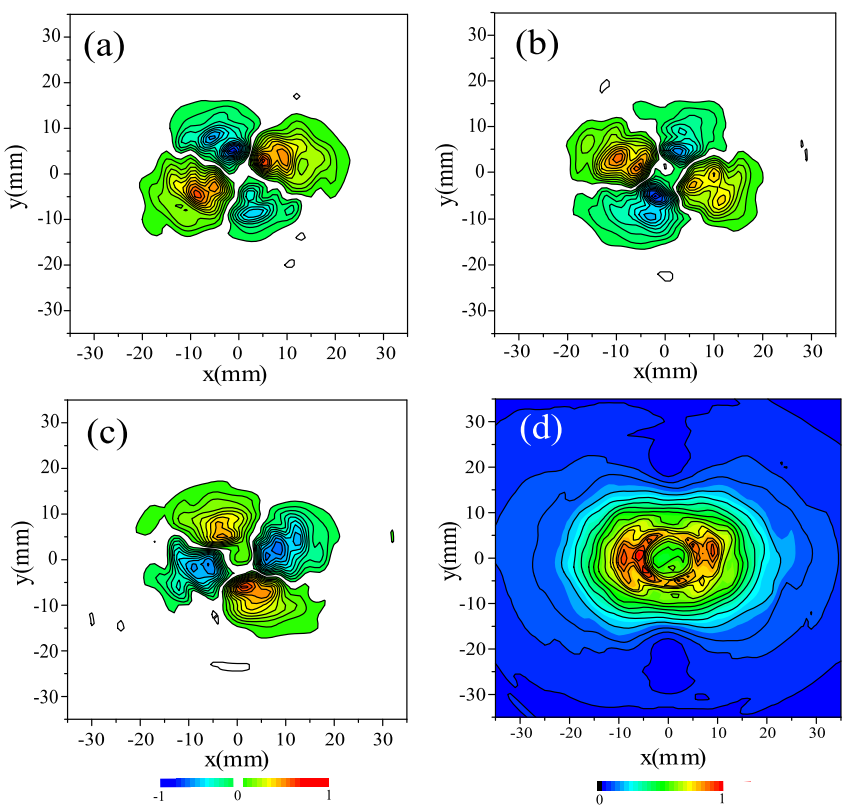

FIG. 5. $y$ component of the electric field $\underline{E}$ of the radiation at different times. (a) $t=0.2$, (b) $t=0.4$, (c) $t=0.6$ ps. (d) Average intensity for $m=2, \varepsilon=1$. 


$$
E_{y}(\underline{x}) \approx \frac{2 e^{2}}{m_{e} c^{2} \gamma} \sum_{j=1}^{N} \int d \underline{x^{\prime}} \mathcal{F}\left(\underline{x}-\underline{x}^{\prime}\right) E_{L}\left(\underline{x}^{\prime}, t_{r}\right) \delta\left(\underline{x}^{\prime}-\underline{x}_{j}\left(t_{r}\right)\right),
$$

with

$$
\mathcal{F}\left(\underline{x}-\underline{x}^{\prime}\right)=\frac{n_{z}\left(n_{z}-\beta_{0}\right)-n_{x}^{2}}{\left(1-\beta_{0} n_{z}\right)^{2}\left|\underline{x}-\underline{x}^{\prime}\right|} .
$$

For putting in evidence the dependence of $E_{y}$ on the angle $\varphi$ of the observer due to the laser structure, which is not explicit in Eq. (9), we introduce a new variable $\rho=\underline{x}-\underline{x}^{\prime}$, that in cylindrical coordinates reads $(\varrho, \psi, \bar{\varsigma})$ and we observe that, within the cone $1 / \gamma, \mathcal{F}(\underline{\rho}) \approx \mathcal{F}(\varrho, \varsigma)$. Moreover, we write the laser field (3), function of $\underline{x}-\underline{\rho}$, as $E_{L}=E_{0} h(r, \varphi, \varrho, \psi) \tilde{f}(z-\varsigma, r-\varrho)$, with

$$
\tilde{f}(z-\varsigma, r, \varrho)=\frac{w_{0}^{2}}{w_{z}^{2}} g\left(z-\varsigma+c t_{r}\right) e^{-\varrho^{2} / 2 w_{z}^{2}} e^{i\left[\omega_{L} t_{r}+k_{z L}(z-\varsigma)\right]},
$$

$$
\begin{aligned}
h(r, \varphi, \varrho, \psi) & =\left(\frac{r}{w_{0}} e^{i \varphi}-\frac{\varrho}{w_{0}} e^{i \psi}\right)^{m} e^{-r^{2} / 2 w_{z}^{2}+\left[r \varrho \cos (\psi-\varphi) / w_{z}^{2}\right]} \\
& =\left(\frac{r}{w_{0}} e^{i \varphi}\right)^{m} e^{-r^{2} / 2 w_{z}^{2}} k(r, \varrho, \psi-\varphi),
\end{aligned}
$$

and

$$
\begin{aligned}
& k(r, \varrho, \psi-\varphi) \\
& \quad=\sum_{k=0}^{m} c_{k, m}\left(\frac{-\varrho}{r} e^{i(\psi-\varphi)}\right)^{k-m} \exp \frac{r \varrho \cos (\psi-\varphi)}{w_{z}^{2}},
\end{aligned}
$$

with $c_{k, m}$ the binomial coefficients. By using the delta function in cylindrical coordinates $\delta\left(\underline{\rho}-\underline{\rho}_{0}\right)=$ $\delta\left(\varrho-\varrho_{0}\right) \delta\left(\psi-\psi_{0}\right) \delta\left(\varsigma-\varsigma_{0}\right) / \varrho$, we obtain

$$
\begin{gathered}
E_{y}(\underline{x})=-\frac{2 e^{2} E_{0}}{m_{e} c^{2} \gamma}\left(\frac{r}{w_{0}} e^{i \varphi}\right)^{m} e^{-r^{2} / 2 w_{z}^{2}} \sum_{j=1}^{N} \int d \varrho d \varsigma d \psi, \\
\delta\left[\underline{x}-\underline{\rho}-\underline{x}_{j}\left(t_{r}\right)\right] \mathcal{F}(\varrho, \varsigma) \tilde{f}(z-\varsigma, r-\varrho) k(r, \varrho, \psi-\varphi) .
\end{gathered}
$$

The field then becomes

$$
\begin{gathered}
E_{y}(\underline{x})=\frac{-2 e^{2} E_{0} r^{m} e^{-r^{2} / 2 w_{z}^{2}}}{m_{e} c^{2} \gamma w_{0}^{m}} \mathrm{e}^{i m \varphi}, \\
\sum_{j=1}^{N} \mathcal{F}\left(r-r_{j}, z-z_{j}\right) \tilde{f}\left(\underline{x}_{j}\right) k\left(r, r-r_{j}, \varphi_{j}\right),
\end{gathered}
$$

where, explicitly,
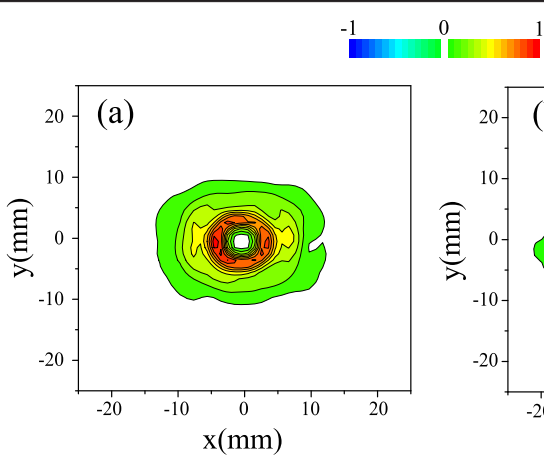

FIG. 6. $z$ component of the angular orbital momentum transverse density $d L_{z}^{\mathrm{OM} \text {,rad }} / d x d y$ of the radiation on a screen at $1 \mathrm{~m}$ for the cases (a) $m=1$ and (b) $m=2$, with the same parameters as Figs. 4 and 5.

$k\left(r, r-r_{j}, \varphi_{j}\right)=\sum_{k=0}^{m} c_{k, m}\left(\frac{r_{j}-r}{r} e^{i \varphi_{j}}\right)^{k-m} e^{\left[r\left(r-r_{j}\right) \cos \left(\varphi_{j}\right) / w_{z}^{2}\right]}$

and its derivative with respect to $\varphi, d E_{y}^{*}(\underline{x}) / d \varphi$ simply is $-i m E_{y}^{*}(\underline{x})$. Finally, by applying definition (7), we have

$$
\frac{d L_{z}^{\mathrm{OM}, \mathrm{rad}}}{d V} \approx \frac{m}{4 \pi \omega}\left|E_{y}\right|^{2} .
$$

The orbital angular momentum turns out to be proportional to $m$ and with sign contrary to the laser momentum. The numerical calculations performed without approximation through Eq. (7), integrated along $z$, for the cases with $m=$ 1 and 2 are presented in Fig. 6, showing the substantial proportionality between the orbital angular momentum and the intensity of the wave and confirming Eq. (15), that is valid within $r \ll L / \gamma$. The total value of the $z$ component orbital momentum per single shot turns out to be $L_{z}^{\mathrm{OM} \text {,rad }}=$ $1.7 \times 10^{-27} \mathrm{~J} \cdot \mathrm{s}$ for $m=1$ and about the double for $m=2$. We can compare this value with the orbital angular momentum variation $\Delta L_{\text {atom }}$ of single atoms involved in electronic transitions with energy variation of about $8 \mathrm{keV}$, close to the photon energy of the radiation (the corresponding wavelength is $\lambda \sim 1.55 \AA$ ). From the applicative point of view, the use of an $x$-ray photon beam carrying angular momentum can allow the study of transitions in high $Z$ materials to states with high order angular momentum, not allowed by ordinary photon beam. In these processes, $\Delta L_{\text {atom }}=\bar{m} \hbar=\bar{m} 1.05 \times 10^{-34} \mathrm{~J} \cdot \mathrm{s}$, where $\bar{m}$ is a low integer number depending on the transition.

In this Letter, we demonstrate that an OAM laser pulse transfers its orbital angular momentum to the x-radiation generated by inverse Compton backscattering with a relativistic electron beam and we calculate the direct relation between the angular orbital momenta of laser and radiation. We propose a scheme of OAM x-ray source based on the inverse Compton scattering between an electron beam and a laser pulse carrying angular 
momentum. The generation of $2.5 \times 10^{6} \mathrm{OAM}$ photons at $8 \mathrm{keV}$ using a linac electron beam at $25 \mathrm{MeV}$, colliding with a Ti:Sapphire laser at $1 \mathrm{~J}$ has been presented. The wide tunability of the Compton sources permits us to produce, with the same laser system, radiation over a large range of frequency with a considerable efficiency.

[1] L. Allen, M. W. Beijersbergen, R. J. C. Spreeuw, and J. P. Woerdman, Phys. Rev. A 45, 8185 (1992).

[2] A. Picón, A. Benseny, J. Mompart, J. R. Vázquez de Aldana, L. Plaja, G. F. Calvo, and L. Roso, New J. Phys. 12, 083053 (2010).

[3] R. Jauregui, Phys. Rev. A 70, 033415 (2004).

[4] M. Babiker, W. L. Power, and L. Allen, Phys. Rev. Lett. 73, 1239 (1994).

[5] A. Alexandrescu, D. Cojoc, and E. I. DiFabrizio, Phys. Rev. Lett. 96, 243001 (2006).

[6] M. Babiker, C. R. Bennett, D. L. Andrews, and L. D. Romero, Phys. Rev. Lett. 89, 143601 (2002).

[7] K. Toyoda, F. Takahashi, S. Takizawa, Y. Tokizane, K. Miyamoto, R. Morita, and T. Omatsu, Phys. Rev. Lett. 110, 143603 (2013).

[8] J. Wang et al., Nat. Photonics 6, 488 (2012).

[9] X. Cai, J. Wang, M. J. Strain, B. Johnson-Morris, J. Zhu, M. Sorel, J. L. O'Brien, M. G. Thompson, and S. Yu, Science 338, 363 (2012).

[10] N. Bozinovic, Y. Yue, Y. Ren, M. Tur, P. Kristensen, H. Huang, A. E. Willner, and S. Ramachandran, Science 340, 1545 (2013).

[11] H. He, M. E. Friese, N. R. Heckenberg, and H. RubinszteinDunlop, Phys. Rev. Lett. 75, 826 (1995).

[12] A. Jesacher, F. Fürhapter, S. Bernet, and M. Ritsch-Marte, Phys. Rev. Lett. 94, 233902 (2005).

[13] J. Verbeeck, H. Tian, and P. Schattschneider, Nature (London) 467, 301 (2010).

[14] M. P. J. Lavery, F. C. Speirits, S. M. Barnett, and M. J. Padget, Science 341, 537 (2013).

[15] F. Tamburini, B. Thidé, G. Molina-Terriza, and G. Anzolin, Nat. Phys. 7, 195 (2011).

[16] S. Carbajo, E. A. Nanni, L. J. Wong, G. Moriena, P. D. Keathley, G. Laurent, R. J. Dwayne Miller, and F. X. Kärtner, Phys. Rev. Accel. Beams 19, 021303 (2016).
[17] A. Picón, J. Mompart, J. R. Vázquez de Aldana, L. Plaja, G. F. Calvo, and L. Roso, Opt. Express 18, 3660 (2010).

[18] M. van Veenendaal and I. McNulty, Phys. Rev. Lett. 98, 157401 (2007).

[19] A. S. Rury, Phys. Rev. A 87, 043408 (2013).

[20] J. Arlt, K. Dholakia, L. Allen, and M. J. Padgett, J. Mod. Opt. 45, 1231 (1998).

[21] A. G. Peele, P. J. McMahon, D. Paterson, C. Q. Tran, A. P. Mancuso, K. A. Nugent, J. P. Hayes, E. Harvey, B. Lai, and I. McNulty, Opt. Lett. 27, 1752 (2002).

[22] B. Terhalle, A. Langner, B. Päivänranta, V. A. Guzenko, C. David, and Y. Ekinci, Opt. Lett. 36, 4143 (2011).

[23] S. Sasaki and I. McNulty, Phys. Rev. Lett. 100, 124801 (2008).

[24] J. Bahrdt, K. Holldack, P. Kuske, R. Müller, M. Scheer, and P. Schmid, Phys. Rev. Lett. 111, 034801 (2013).

[25] E. Hemsing, A. Marinelli, and J. B. Rosenzweig, Phys. Rev. Lett. 106, 164803 (2011).

[26] E. Hemsing, A. Knyazik, M. Dunning, D. Xiang, A. Marinelli, C. Hast, and J. B. Rosenzweig, Nat. Phys. 9, 549 (2013).

[27] E. Hemsing and A. Marinelli, Phys. Rev. Lett. 109, 224801 (2012).

[28] P. R. Ribič, D. Gauthier, and G. De Ninno, Phys. Rev. Lett. 112, 203602 (2014).

[29] U. D. Jentschura and V. G. Serbo, Phys. Rev. Lett. 106, 013001 (2011).

[30] U. D. Jentschura and V. G. Serbo, Eur. Phys. J. C 71, 1571 (2011).

[31] S. Stock, A. Surzhykov, S. Fritzsche, and D. Seipt, Phys. Rev. A 92, 013401 (2015).

[32] J. D. Jackson, Classical Electrodynamics, 3rd ed. (Wiley, New York, 1998).

[33] G. Dattoli, J. Math. Anal. Appl. 284, 447 (2003); V. Petrillo et al., Optical beam with vortices: a first order paraxial analysis, PAHBB Conference 2016, submitted to NIMA_ procedings.

[34] P. Oliva, A. Bacci, U. Bottigli, M. Carpinelli et al., Nucl. Instrum. Methods Phys. Res., Sect. A 615, 93 (2010).

[35] C. Vaccarezza et al., Nucl. Instrum. Methods Phys. Res., Sect. A 829, 237 (2016).

[36] V. Petrillo et al., Nucl. Instrum. Methods Phys. Res., Sect. A 693, 109 (2012).

[37] V. Petrillo et al., Phys. Rev. ST Accel. Beams 18, 110701 (2015). 